\title{
Format-dependent representations of symbolic and non-symbolic numbers in the human cortex as revealed by multi-voxel pattern analyses
}

\author{
J. Bulthéa,b, B. De Smedtt,*, H. Op de Beeck ${ }^{a, *}$ \\ ${ }^{a}$ Laboratory of Biological Psychology, University of Leuven (KU Leuven), Tiensestraat \\ 102, B-3000 Leuven, Belgium \\ ${ }^{b}$ Parenting and Special Education Research Unit, University of Leuven (KU Leuven), \\ Leopold Vanderkelenstraat 32, B-3000 Leuven, Belgium
}

\begin{abstract}
Neuroimaging studies in the last 20 years have tried to unravel the neural correlates of the number processing across formats in humans and nonhuman primates. Results point to the intraparietal sulcus as the core area for an abstract representation of numerical quantity. On the other hand, there exists a variety of behavioral and neuroimaging data that are difficult to reconcile with the existence of such an abstract representation. In this study; we addressed this issue by applying multivoxel pattern analysis (MVPA) to functional Magnetic Resonance Imaging (fMRI) data to unravel the neural representations of symbolic (digits) and non-symbolic (dots) numbers and their possible overlap on three different spatial scales (entire lobules, smaller regions of interest and a searchlight analysis with 2 -voxel radius). Results showed that numbers in both formats are decodable in occipital, frontal, temporal and parietal regions. However, there were no overlapping representations between dots and digits on any of the spatial scales. These data suggest that the human brain does not contain an abstract representation of numerical magnitude.
\end{abstract}

Keywords: Numerical cognition, fMRI, Multi-voxel pattern analysis, parietal cortex, numbers

\footnotetext{
*Corresponding author

Email addresses: bert.desmedt@ppw.kuleuven.be (B. De Smedt), hans.opdebeeck@ppw.kuleuven.be (H. Op de Beeck)
} 


\section{Introduction}

The neural mechanisms of numerical cognition have been intensively investigated in behavioral and neuroimaging research (for review see Ansari (2008); Nieder \& Dehaene (2009)) due to the central role of numbers in daily life and education (Gerardi et al., 2013; Lipkus \& Peters, 2009 ; Nelson et al., 2008; Reyna et al., 2009; Zikmund-Fisher et al., 2007). A core theme in this research deals with the question of representational overlap between symbolic (e.g. Arabic digits) and non-symbolic (e.g. arrays of dots) magnitudes. This issue has been approached by comparing the brain activity during non-symbolic as well as symbolic tasks and by searching for regions that are commonly active while processing these two formats of numerical magnitudes. Both approaches have provided evidence in favor of the existence of an abstract representation of numerical magnitudes and the accumulating evidence suggests that the intraparietal sulcus (IPS) hosts a core module for processing numerical magnitude (Dehaene \& Cohen, 1997, Eger et al., 2003, Pinel et al., 2001a).

More recently, it has been suggested that the multi-voxel pattern analysis (MVPA) of fMRI data might be an interesting method to probe the abovementioned question. This method provides a more fine-grained understanding of the nature of the activated numerical representations (Raizada et al., 2010). The existing body of data that has been interpreted in favor of an abstract representation of numerical magnitude is typically based on null results, indicating no differences between symbolic and non-symbolic formats in behavioral tasks and in activity in the IPS. Such null results are, however, difficult to interpret, as they can occur due to insufficient power to detect a difference. The present study attempts to overcome this issue by applying MVPA of fMRI data on regions of interest (ROI) throughout the entire cortex (a) to test if symbolic and non-symbolic numerical magnitudes are processed in the same brain areas, and (b) to investigate the amount of representational overlap between both formats in those brain areas. Although MVPA analyses have been applied to investigate numerical processing (Damarla \& Just, 2012, Eger et al., 2009; Raizada et al., 2010), the present study extends the existing body of evidence in two important ways. First, this study is the first to apply MVPA not only in the IPS but also outside the parietal cortex. This allowed us to test the existence of a format-independent system for representing numerical magnitudes. Second, we also used MVPA searchlight analysis in the whole cortex to uncover other possible (common) areas for processing symbolic and non-symbolic magnitudes. 
There has been a longstanding behavioral tradition in attempting to reveal the common representation of different numerical formats (Barth et al., 2003 , Buckley \& Gillman, 1974; Dehaene \& Akhavein, 1995; Jaffe-Katz et al., 1989, Naccache \& Dehaene, 2001; Shepard et al., 1975). One of the most important findings is the so-called numerical distance effect (Moyer \& Landauer, 1967). The numerical distance effect is the observation that reaction times increase and accuracy rates decreases in number comparison tasks when numerical magnitudes are closer in distance than when they are further apart. This effect has been observed in children (Feigenson et al., 2004; Lonnemann et al. 2011; Holloway \& Ansari, 2010; Sekuler \& Mierkiewicz, 1977), adults (Dehaene et al., 1990; Dehaene, 1992; Moyer \& Bayer, 1976; van Opstal \& Verguts, 2011) and animals (Brannon et al., 2001; Nieder \& Miller, 2003) and it has been contended that this indicates a similar way of representing numerical magnitudes across different species and ages. Moreover, this numerical distance effect seems to be similar for symbolic and non-symbolic stimuli, which suggests a common numerical magnitude system for different formats (Dehaene et al., 1990).

Neuroimaging studies in the last 20 years have tried to unravel the neural correlates of this numerical distance effect and number processing across formats in humans and non-human primates. Results have pointed to the IPS as the core area for the representation of numerical magnitude because of three main findings: (a) the IPS is involved in magnitude processing in humans (for a meta-analysis and review see Ansari (2008); Nieder \& Dehaene (2009) ) and primates (Nieder et al., 2002, Nieder \& Miller, 2003; Sawamura et al., 2002); (b) the IPS activity correlates with the distance between numerical magnitudes in humans (Ansari et al., 2006, Cohen Kadosh et al., 2005, Piazza et al., 2007, Pinel et al., 2004); (c) the IPS activity does not differ between formats in humans (Eger et al., 2003; Fias et al., 2003; Piazza et al., 2007).

Although these findings have been replicated with different paradigms and tasks, the abstract processing of numerical magnitudes and the function of the IPS as number module remain a debated issue. More specifically, some behavioral and neuroimaging observations are very difficult to reconcile with the abstract view on magnitude processing (for extensive discussion, see Cohen Kadosh \& Walsh (2009)). For example, Gebuis \& Reynvoet (2012a) have shown that the processing of non-symbolic magnitude is more grounded in low-level visual parameters than the processing of symbolic quantities. Lyons et al. (2012) found that comparing numerical magnitudes across formats is more difficult than comparisons within one format, suggesting that additional processing is required for cross-format comparisons. 
The experiments of Maloney et al. (2010) demonstrated that the distance effect of non-symbolic magnitudes is not correlated with that of symbolic magnitudes. Furthermore, children with developmental dyscalculia are more impaired in symbolic tasks compared to non-symbolic tasks (De Smedt \& Gilmore, 2011; Iuculano et al., 2008; Landerl \& Kölle, 2009; Rousselle \& Noël, 2007). Finally, a recent review by De Smedt et al. (2013) on the association between numerical magnitude processing and individual differences in mathematics achievement indicated that in typically developing children, measures of symbolic but not non-symbolic number processing are reliable predictors of individual differences in mathematics achievement (De Smedt et al.). In sum, these behavioral data are difficult to reconcile with an abstract representation of numerical magnitudes.

This abstract representation of numerical magnitudes has also been challenged by patient and neuroimaging studies. A study on patients with damage to the left supramarginal gyrus showed a dissociation between the processing of symbolic and non-symbolic magnitudes (Polk et al., 2001). Neuroimaging studies have demonstrated that the IPS contains an abstract representation of numerical order rather than numerical magnitude (Fias et al., 2007; Ischebeck et al., 2008) and that activity in the IPS while performing a numerical task was related to response-selection rather than numerical processing per se (Cappelletti et al., 2010, Göbel et al., 2004).

Whether or not numerical magnitudes are processed in an abstract way in the IPS has been subject to a continuing discussion in the numerical cognition domain (Cohen Kadosh \& Walsh, 2009). One of the main issues in this debate is the fact that evidence for an abstract representation of numerical magnitudes is based on null results, indicating no differences across formats in activation in the IPS. It is crucial to point out that these null results emerge from fMRI studies that have used univariate methods to measure the overall regional activity for different conditions. Such data, however, limit our understanding of the information encoded by neural populations in that region. Recently, it has been suggested that the application of MVPA to fMRI might be one way to solve this issue (Ansari, 2008, Cohen Kadosh \& Walsh, 2009; Dehaene, 2009).

MVPA allows to identify spatial patterns of brain activity of different stimuli in a certain region of interest (Norman et al., 2006). Two previous studies have used MVPA to relate the processing of symbolic and non-symbolic formats directly to each other. Damarla \& Just (2012) showed that the neural codes for quantities of objects, e.g., a picture of three tomatoes or the digit 3 with a picture of one tomato, can be accurately decoded in the parietal cortex. Eger et al. (2009) compared the activation patterns evoked 
by dot patterns and digits (numbers 2, 4, 6 and 8) in the parietal cortex. The activation patterns non-symbolic and symbolic magnitudes were distinguishable at the individual level and they could be significantly decoded in the parietal cortex. However, the decoding was less accurate for symbolic compared to non-symbolic magnitudes. Eger et al. (2009) also applied cross-format generalization, showing significant generalization from symbolic to non-symbolic magnitudes but not from non-symbolic to symbolic. The studies of Damarla \& Just (2012) and Eger et al. (2009) demonstrated that MVPA has the sensitivity that is required to investigate the representations of magnitudes. These data also suggested at least some communality in symbolic and non-symbolic representations of magnitudes.

Similar to the study of Eger et al. (2009) we investigated the representation of numerical magnitude in the context of a comparison task. However, we extended their design in three important ways. First, given the growing literature, which shows that non-symbolic comparison tasks involve a lot of non-numerical processes (Gilmore et al., 2013; Gebuis \& Reynvoet, 2012b a), we implemented a whole-brain approach to define the relative importance of the different lobules. Our approach consisted of including a large set of ROIs and searchlight analysis. In these analyses we targeted the neural representations of number at multiple spatial scales: a large spatial scale (the entire cortex, frontal, parietal, occipital and temporal lobes), an intermediate spatial scale (ROIs in the four cortices) and small scale (a whole-brain searchlight analysis with a radius of twice the voxel size ). Second, Eger et al. (2009) only used ten subjects, but we aimed to replicate this in a larger sample of 16 subjects. Third, the paradigm of Eger et al. (2009) was an event-related fMRI design in which each trial involved the presentation of a sample number followed by a match number. Participants had to indicate whether the match number was smaller or larger than the sample number. We opted for a fixed comparison task in which each of the numbers $(2,4$, 6 and 8 ) had to be compared to the fixed reference number 5 and control in this way for possible context-dependent effects on the number representations. The consequence of this fixed comparison task, is that different from Eger et al. (2009) we cannot look into the neural representations of numerical magnitudes without the context of a comparison task.

We expected accurate decoding performance for both symbolic and nonsymbolic magnitudes. If this decoding would be limited to the IPS, this would favor the existence of a format-independent module for representing numerical magnitudes. On the other hand, if decoding performance would be observed across various brain areas, this would suggest that the representation of magnitudes would be more widely distributed throughout the brain. 
We also predicted that a neural distance effect would occur for both formats in the regions with accurate decoding. The decoding performance for small distances should be lower than for large distances. Finally, we tested the generalization between the two numerical formats. Such generalization should occur if there is an abstract representation of number. However, the absence of generalization between the two formats would indicate that there is no such abstract representation of numerical magnitude.

\section{Materials \& Methods}

\subsection{Subjects}

Sixteen healthy subjects participated in the fMRI study (four males, aged between 21-28 years, two left-handed) and were paid for participation. All participants had normal or corrected-to-normal vision and reported no neurological or psychiatric history. No effects of handedness or sex were observed. The study was approved by the medical ethics committee of the KU Leuven. All participants provided informed consent prior to scanning.

\subsection{Stimuli}

Images (400 x 400 pixels) consisted of centered white circles on a black background. Within the white circles either dots or Arabic digits were displayed comprising 2, 4, 6 or 8 as numerosities. Dot displays were controlled for possible confounding parameters, such as intensive parameters (individual item size and inter-item spacing) and extensive parameters (total luminance and total area spanned by the dots). This was done by using the method and automated program created by Dehaene et al. (2005), similar to many other fMRI-studies that used dot patterns as their stimuli (Eger et al., 2009; Roggeman et al., 2011; Santens et al., 2010). Both intensive and extensive parameters were randomly varied across the dot displays by an adapted version of a Matlab program (Matlab 7.13.0, The MathWorks, Inc., Natick, MA) described in Dehaene et al. (2005). To avoid adaptation for the symbolic numbers, the symbols varied in position and size across trials.

\subsection{Apparatus}

The fMRI data were acquired in a 3T Philips Intera Scanner (Department of Radiology of KU Leuven) with a 12-channel head coil and with an EPI sequence (48 slices, $2.12 .1 \mathrm{~mm}$ inplane voxel size, slice thickness 2 $\mathrm{mm}$, interslice gap $1 \mathrm{~mm}$, repetition time $(\mathrm{TR})=3000 \mathrm{~ms}$, echo time (TE) 
$=30 \mathrm{~ms}$, flip angle $=90,104104$ matrix). Accurate timing of the stimuli relative to fMRI acquisition was achieved with an electronic trigger at the beginning of each run. For each participant, a high-resolution T1-weighted anatomical image was obtained (182 slices, resolution $0.98 \times 0.98 \times 1.2 \mathrm{~mm}$, $\mathrm{TR}=9.6 \mathrm{~ms}, \mathrm{TE}=4.6 \mathrm{~ms}, 256256$ acquisition matrix).

Stimulus presentation in all tasks was controlled via Psychtoolbox 3 (Brainard, 1997). We used a Barco 6400i LCD projector (resolution 1024 768, refresh rate $75 \mathrm{~Hz}$ ) to project the stimuli on a vertical screen. The stimuli were positioned approximately $35 \mathrm{~cm}$ from participants' eyes, and were visible via a mirror attached to the head-coil.

\subsection{Design}

The design of the experimental runs is illustrated in Figure 1. We used a short-block design with variable block duration, i.e. one block comprised either 4, 5 or 6 seconds. Each run consisted of 48 experimental blocks (each condition was presented in 6 blocks) and 7 fixation blocks (baseline). Two fixation blocks were presented for 8 seconds at the beginning and at the end of the run, the remaining five fixation blocks lasted 4,5 or 6 seconds and were presented after each 8th experimental block. During each experimental block, one particular number was repeated in the same format in sequences of 4, 5 or 6 trials. Each trial was presented for $1000 \mathrm{~ms}$, during which a random exemplar of the number was shown for $200 \mathrm{~ms}$ and followed by a fixation cross for $800 \mathrm{~ms}$. In total, one run lasted 280 seconds and 12-14 runs were presented to participants (Figure 1).

Participants had to perform a number comparison task in the experimental runs. We selected this task because it allowed us to explicitly access the numerical magnitude representations (Piazza et al., 2004, Pinel et al., 2004, Zorzi et al., 2011). Participants were instructed to evaluate whether the presented number was smaller or larger than five whenever the displayed format or numerosity changed.

In two localizer runs, we used a subtraction task. In this task participants had to subtract two numbers ranging from 1 to 20 and they had to indicate whether the solution was odd or even. In each trial the subtraction problem was presented for $1700 \mathrm{~ms}$ and followed by a fixation cross for $300 \mathrm{~ms}$.

\subsection{Analysis}

\subsection{1. fMRI preprocessing}

The data were processed with the Statistical Parametric Mapping software package (SPM8, Welcome Department of Cognitive Neurology, London), as well as custom Matlab code. Anatomical images were normalized 


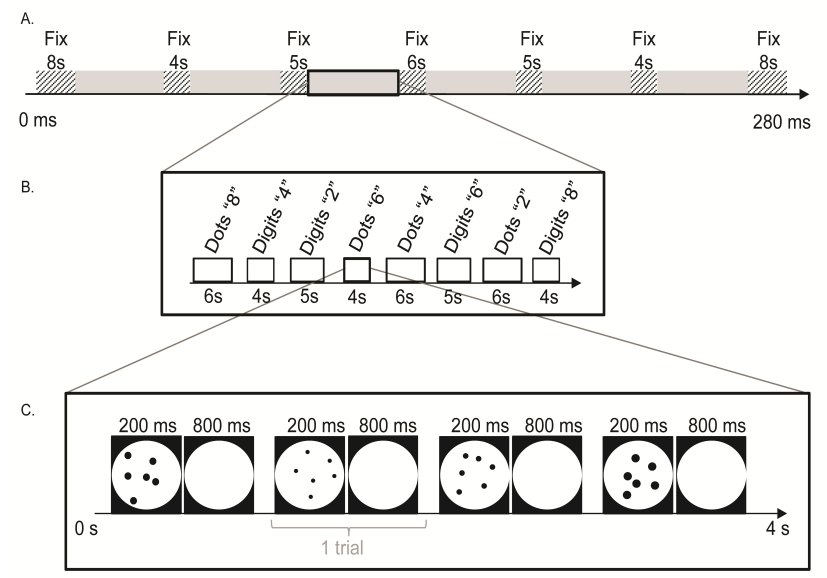

Figure 1: Overview of the experimental design; a. One run lasted 280 seconds and consisted of 7 fixation blocks between the experimental blocks; b. Example of 8 experimental blocks; c. Example of one experimental block comprising 4 trials.

to the standard brain template defined by the Montreal Neurological 152brains average. Functional images were corrected for slice-timing differences and realigned to the first image to correct for head movements. Spatial normalization was done using the parameters obtained in the normalization of the anatomical images. During normalization functional images were resampled to a voxel-size of $2 \times 2 \times 2 \mathrm{~mm}$. Finally functional images were spatially smoothed using Gaussian kernels of $4 \mathrm{~mm}$ full-width at half maximum (FWHM). It could be that spatial smoothing affects the decoding analyses, although Op de Beeck et al. (2010) observed that smoothing does not decrease the sensitivity of decoding analyses. Against this background, we tested whether there was a significant difference between the decoding accuracy of smoothed and unsmoothed data. These data revealed no significant differences between the regions of interest (decoding symbols: $F_{(1,22)}=$ $0.513, p=0.48$ and decoding dots: $\left.F_{(1,22)}=0.028, p=0.87\right)$, which corresponds to Op de Beeck et al. (2010).

\subsubsection{Statistical analysis}

The experimental effects in each voxel were estimated by a multi-session design matrix that modeled the data at the block level. A general linear model was created with regressors for each participant for each condition. The six motion realignment parameters were additionally included as regres- 
sors to account for signal variations due to head movements. Subsequent analyses were performed using t-statistics (resulting from the contrast of each condition versus baseline) per run that were obtained after fitting the general linear model.

\subsubsection{Regions of Interest}

Twenty-three ROIs were defined on a subject level using the functional data from the localizer scans (contrast of task minus fixation) and the anatomical WFU PickAtlas Toolbox (Wake Forrest University PickAtlas, http://fmri.wfubmc.edu/cms/software) when the ROI was available in the toolbox (Maldjian et al., 2003). The selection of ROIs was theoretically guided by including ROIs that have been reported to involved in numerical processing (Dehaene et al., 2003; Holloway et al., 2013, Lyons \& Ansari, 2009; Maruyama et al., 2012; Piazza et al., 2007; Santens et al., 2010; Zago et al., 2001; Zhang et al., 2012).

We selected the ROIs on different spatial scales: (a) large spatial scale (the entire cortex, frontal, parietal, temporal, occipital cortex, frontal + parietal + temporal cortex and frontal + parietal cortex); (b) intermediate spatial scale (IPS, left and right superior parietal lobule, inferior occipital cortex, superior temporal sulcus, visual word form area, Wernickes area, fusiform gyrus, left and right inferior frontal gyrus, and left and right superior frontal gyrus; (c) small spatial scale (left and right anterior IPS, and left and right posterior IPS). The contrast for defining the ROIs was thresholded at a minimum of $p<0.001$ (uncorrected for the number of voxels). The All Regions ROI contained all the voxels that resulted from the localizer runs in each subject.

\subsubsection{Pattern classification}

We implemented decoding and generalization pattern classification analysis for every ROI by applying linear support vector machines (SVM) using the OSU SVM Matlab toolbox with the default parameters of this toolbox as described by Op de Beeck et al. (2010). We used a radial basis function kernel as decision function with the parameter gamma set to 1 . The type of the classification algorithm was C-SVC with $\mathrm{C}$ set to 1 . During the training of the linear SVM $70 \%$ of the data was used to construct the hyperplane that best separated the data of the two conditions. The performance of the classifier on this pair-wise classification was calculated for the average data of the remaining $30 \%$ of the runs (repeated 100 times per pair of conditions with a random assignment of runs to the training and test sets). For decoding pattern classification, the pair-wise classification was performed on 
the same condition pairs during training and testing. The higher the decoding accuracy, the better the classifier was able to discriminate between two conditions. For generalization pattern classification, different condition pairs were used to train versus test the performance of the classifier. A higher accuracy for the generalization analyses suggests an overlap in the neural representations for the training conditions and the test conditions. We also performed analyses with correlation-based MVPA instead of linear classifiers, which resulted in similar conclusions as the decoding accuracies.

\subsubsection{Distance effect analysis}

To test for a neural distance effect, we examined how the pairwise classification results varied with the numerical distance between the two classified numerical magnitudes. We applied a regression analysis in Matlab with the three possible distances $(2,4$ and 6$)$ as predictors and the decoding classification performance for that distance as the dependent variable for those ROIs that showed a significant decoding effect.

\subsubsection{Searchlight analysis}

The Searchlight Analysis (SLA) is a MVPA method introduced as a technique for localizing functional regions that carry out a particular type of information. This method particularly suited for finding where in the brain the local spatial activity pattern differs across conditions (Kriegeskorte et al., 2006, Kriegeskorte \& Bandettini, 2007). These analyses are performed at a smaller spatial scale and therefore, they nicely complement the MVPA on large (entire cortex and lobes) and intermediate (ROIs) spatial scales.

For the SLA a sphere with a radius of twice the voxel size (which includes 33 voxels, if all the surrounding voxels are within the brain volume) was sequentially moved across the entire volume (non-brain volumes areas were left out of the analysis). White matter was included in the resulting maps of decoding accuracy, generalization accuracy and neural distance effect for each subject. Afterwards the maps were spatially smoothed using Gaussian kernels of $6 \mathrm{~mm}$ FWHM. These output images of all subjects were submitted to a second-level random effects analysis with SPM8. In this analysis, the average accuracy of pattern classification tests for each voxel was compared to chance level (0.50) and the group t-map that contains the corresponding t-value for each voxel was generated (Lee et al., 2011; Raizada et al., 2010, Walther et al., 2009). To correct for multiple comparisons the false discovery rate was set at $p=0.05$. 


\section{Results}

\subsection{Behavioral Results}

A two-way repeated-measures (distance $\mathrm{x}$ format) ANOVA was performed on the accuracy and reaction times of the number comparison task for symbolic and non-symbolic magnitudes. For accuracy, there was significant main effect of distance $\left(F_{(1,15)}=15.24, p=0.001\right)$ suggesting more accurate responses for larger distances than for smaller distances. There was no main effect of format $\left(F_{(1,15)}=1.004, p=0.332\right)$ and no distance $\mathrm{x}$ format interaction $\left(F_{(1,15)}=0.017, p=0.897\right)$.

Turning to the reaction times, we found a significant main effect of distance $\left(F_{(1,15)}=90.629, p<0.001\right)$. Participants responded faster to larger distances than to smaller distances. There was also a significant main effect of format $\left(F_{(1,15)}=83.457, p<0.001\right)$, with faster reaction times for symbolic magnitudes than for non-symbolic magnitudes, and a significant distance $\mathrm{x}$ format interaction $\left(F_{(1,15)}=38.575, p<0.001\right)$ which indicated a larger distance effect for non-symbolic magnitudes compared to symbolic magnitudes. To examine the association between the behavioral symbolic and non-symbolic distance effects, we calculated for each participants a linear regression in which the reaction times for each task was predicted by distance. $R^{2}$-squared values for each regression were used as indeces of the distance effect. No significant correlation between the distance effects for symbolic and nonsymbolic magnitudes was observed $(r=0.108, p=0.69)$.

\section{2. fMRI Results}

\subsubsection{Localizer}

Participants had an average accuracy of $85.93 \%(\mathrm{SD}=6.26)$ on the subtraction task. A second-level analysis on the fMRI localizer data was applied to the contrast 'subtraction task minus fixation' for all the subjects. We observed significant activation in multiple regions across the entire cortex, including the IPS, inferior and superior frontal gyri, supramarginal and angular gyri, inferior occipital cortex and superior parietal cortex. Results are shown in Figure 2 and Table 1.

\subsubsection{Symbolic and non-symbolic representations: large spatial scale (entire cortex and lobes)}

We first investigated if symbolic and non-symbolic representations, distance effects for both formats and an the association between digits and dots can be detected on a large spatial scale. 


\begin{tabular}{lccccc}
\hline ROI & $\mathrm{x}$ & $\mathrm{y}$ & $\mathrm{z}$ & $t[15]$ & $p$ \\
\hline Left supramarginal angular & -8 & 8 & 60 & 13.32 & $<0.001$ \\
Left precentral gyrus & -50 & 5 & 28 & 13.16 & $<0.001$ \\
hIP3 & -22 & -55 & 40 & 11.76 & $<0.001$ \\
Left inferior occipital cortex & -44 & -86 & -8 & 10.62 & $<0.001$ \\
Right superior parietal cortex & 24 & -55 & 50 & 10.12 & $<0.001$ \\
Left superior parietal cortex & -22 & 66 & 44 & 10.02 & $<0.001$ \\
Right inferior occipital cortex & 42 & -84 & -8 & 9.15 & $<0.001$ \\
Right IPS & 32 & -52 & 46 & 8.83 & $<0.001$ \\
Right fusiform gyrus & 44 & -76 & -18 & 8.46 & $<0.001$ \\
Left IPS & -44 & -44 & 42 & 8.45 & $<0.001$ \\
Left fusiform gyrus & -46 & -62 & -20 & 7.89 & $<0.001$ \\
Right superior frontal gyrus & 32 & 4 & 64 & 5.59 & 0.002 \\
Right inferior frontal gyrus & 48 & 10 & 28 & 5.23 & 0.003 \\
Left insula & -26 & 24 & 2 & 5.01 & 0.003 \\
Right middle frontal gyrus & 44 & 0 & 54 & 4.97 & 0.004 \\
Left superior frontal gyrus & -20 & 8 & 70 & 4.94 & 0.004 \\
Left inferior frontal gyrus & -32 & 28 & -4 & 4.2 & 0.011 \\
Left middle frontal gyrus & -42 & 38 & 28 & 4.05 & 0.013 \\
Right precentral gyrus & 42 & 2 & 40 & 3.27 & 0.013 \\
\hline
\end{tabular}

Table 1: List of regions activated by the localizer subtraction task. Multiple comparison correct with FDR $=0.05$. The $\mathrm{x}, \mathrm{y}, \mathrm{z}$ coordinates are the MNI-coordinates of each activation peak. hIP3 is a cytoarchitectonic defined subregion of the IPS (Caspers et al. 2006). 


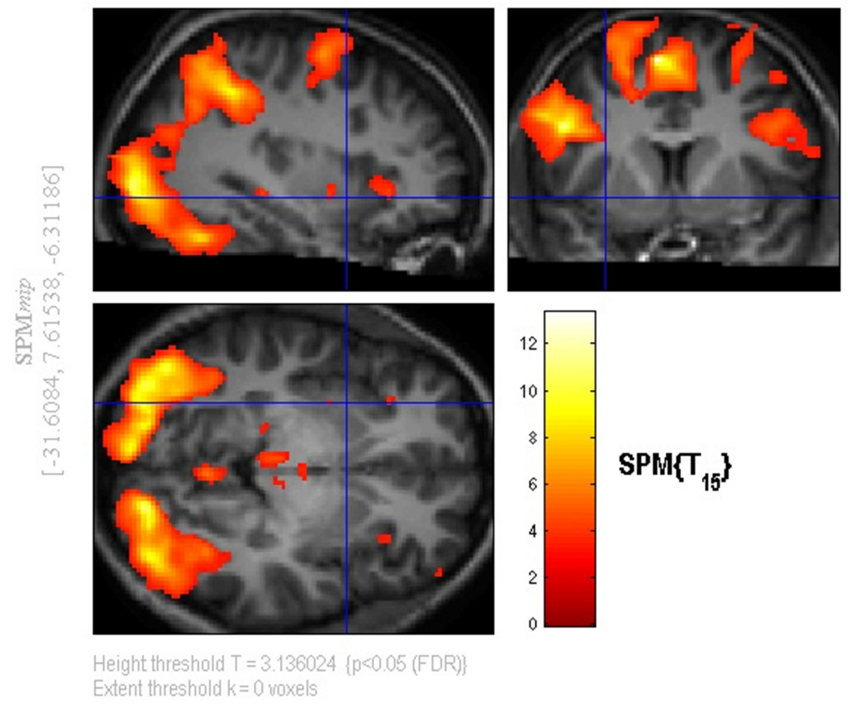

Figure 2: Statistical map of brain regions that were found to exhibit significantly higher levels of activations during the subtraction task compared to fixation (multiple comparisons corrected with FDR $=0.05$, mapped onto an anatomical image of one of the participants).

Decoding accuracies. For both non-symbolic and symbolic magnitudes the classifier was able to discriminate activation patterns of different magnitudes significantly better than chance level in the entire cortex, frontal cortex, parietal cortex, occipital cortex, frontal + parietal + temporal cortex, and frontal + parietal cortex (Figure 3). These data indicate that the neural representations of numbers for both formats seem to be present on a large spatial scale and in all cortices. 


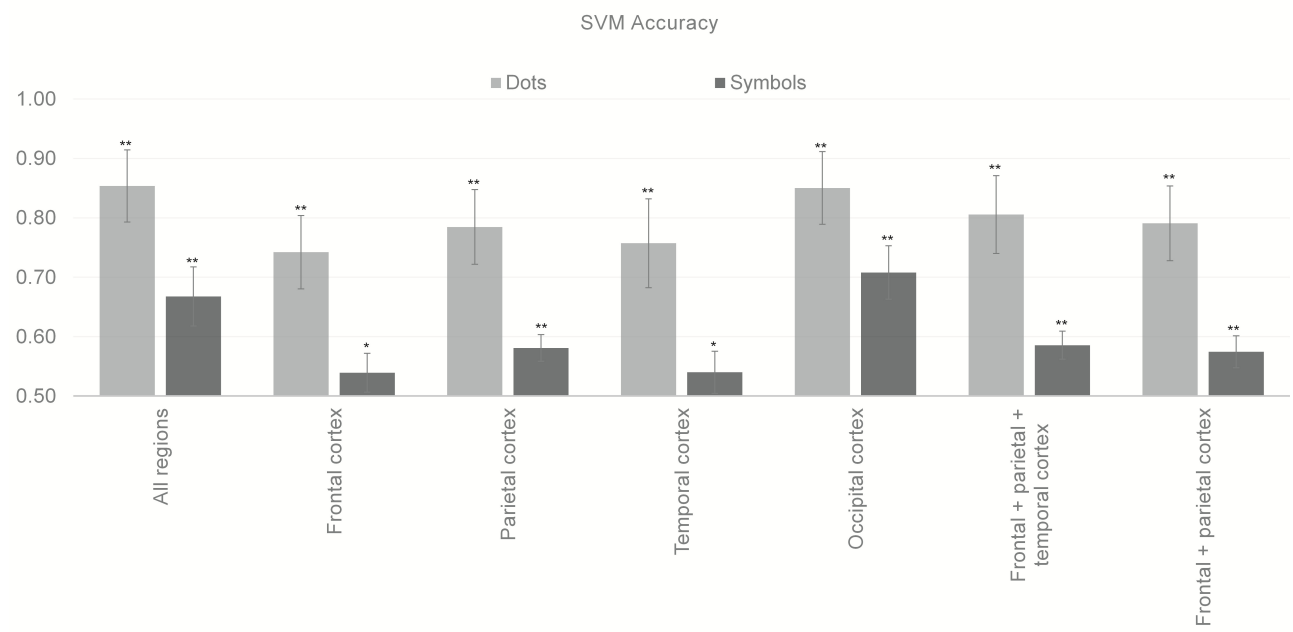

Figure 3: Significant decoding performances for symbolic and non-symbolic stimuli for the cortices, the error bars represent the standard error of mean $(* p<0.05 * * p<0.01)$.

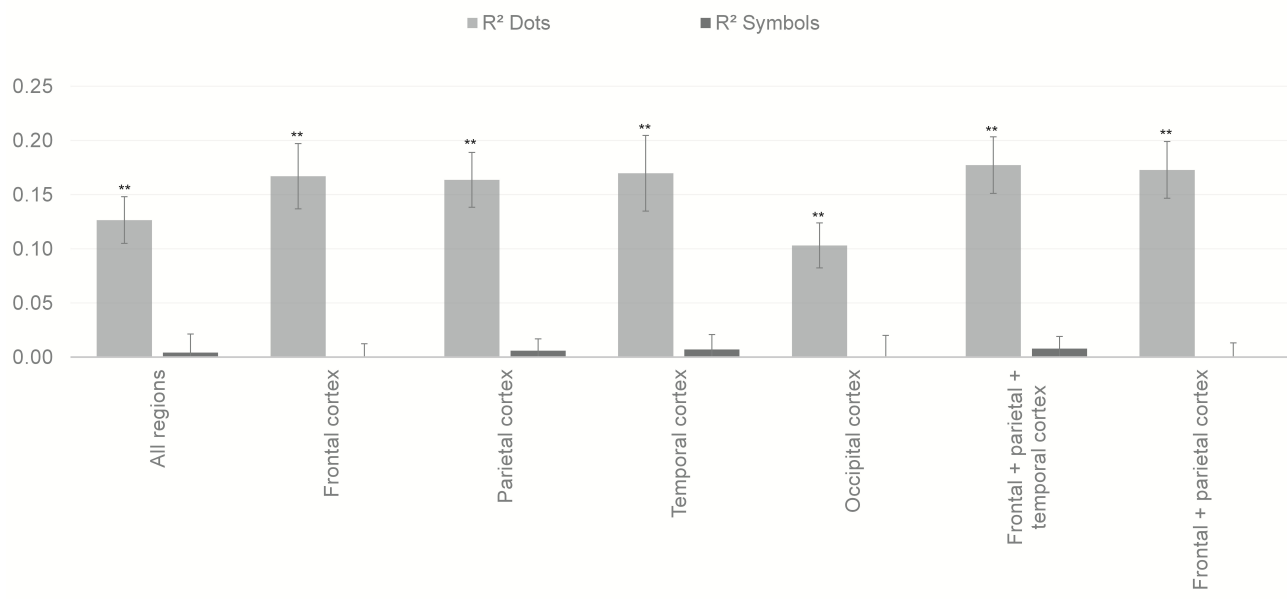

Figure 4: The distance effect of dots and symbols in the different cortices. The error bars represent the standard estimate of error $\left({ }^{*} p<0.05{ }^{* *} p<0.01\right)$. 
Neural distance effect. To test whether the significant decoding of symbolic and non-symbolic magnitudes on a large spatial scale was due to the underlying magnitude of the stimuli, we applied a regression analysis to the decoding accuracies of those ROIs that showed significant decoding performance (Figure 4). For non-symbolic magnitudes all the cortices showed a significant distance effect: All regions $\left(R^{2}=0.13, p<0.001\right)$, frontal cortex $\left(R^{2}=0.17, p<0.001\right)$, parietal cortex $\left(R^{2}=0.16, p<0.001\right)$, temporal cortex $\left(R^{2}=0.0 .17, p<0.001\right)$, occipital cortex $\left(R^{2}=0.10, p=0.002\right)$, frontal + parietal + temporal cortex $\left(R^{2}=0.18, p<0.001\right)$ and parietal + frontal cortex $\left(R^{2}=0.17, p<0.001\right)$. These results indicate that we captured magnitude related activity for non-symbolic magnitudes on a large spatial scale.

For symbolic magnitudes, there was no distance effect in any of the cortices: All regions $\left(R^{2}=0.004, p=0.54\right)$, frontal cortex $\left(R^{2}=0.0003, p=0.87\right)$, parietal cortex $\left(R^{2}=0.01, p=0.47\right)$, temporal cortex $\left(R^{2}=0.01, p=\right.$ $0.43)$, occipital cortex $\left(R^{2}=0, p=0.96\right)$, frontal + parietal + temporal cortex $\left(R^{2}=0.01, p=0.41\right)$ and parietal + frontal cortex $\left(R^{2}=0.0004, p\right.$ $=0.85)$. This all suggests that for symbolic magnitudes we did not capture any magnitude related activity on a large spatial scale.

Association digits and dots. To investigate the invariance of the representation of magnitude across formats, we applied generalization pattern classification. For this, we trained the classifier to discriminate between two magnitudes in one format (for example between symbol 2 and symbol 4) and tested the performance of the classifier on discriminating between the same magnitudes in the other format (i.e., 2 dots and 4 dots). If a ROI contains an abstract representation of magnitude, one would expect that this representation gereralizes across different formats. The results for this analysis are summarized in Table 2 .

There was no significant generalization from non-symbolic to symbolic magnitudes or from symbolic to non-symbolic magnitudes in any of the lobes, suggesting no representational overlap between both formats on a large spatial scale.

\subsubsection{Symbolic and non-symbolic representations: relevant ROIs in the lobes}

To look further within the cortices, we selected a series of ROIs in the different lobes based on previous numerical cognition literature (see Methods, Regions of Interest). This was done to test whether numerical magnitude representations, a neural distance effect and a possible association between 


\begin{tabular}{lcccccc}
\hline ROI & \multicolumn{3}{c}{ Dots to digits } & \multicolumn{3}{c}{ Digits to dots } \\
\hline & Accuracy & $t[15]$ & $p$ & Accuracy & $t[15]$ & $p$ \\
All Regions & 0.49 & -1.20 & 0.87 & 0.51 & 0.51 & 0.31 \\
Frontal, Parietal \& Temporal Cortex & 0.49 & -0.94 & 0.87 & 0.52 & 1.14 & 0.14 \\
Frontal \& Parietal Cortex & 0.49 & -0.87 & 0.80 & 0.50 & -0.02 & 0.51 \\
Frontal Cortex & 0.50 & -0.33 & 0.63 & 0.50 & 0.13 & 0.45 \\
Parietal Cortex & 0.49 & -1.12 & 0.86 & 0.49 & -0.70 & 0.75 \\
Temporal Cortex & 0.50 & 0.09 & 0.46 & 0.51 & 0.67 & 0.26 \\
Occipital Cortex & 0.49 & -0.94 & 0.82 & 0.51 & 1.02 & 0.16 \\
\hline
\end{tabular}

Table 2: Overview of the generalization results for generalization from dots to digits and from digits to dots.

dots and digits can be observed on an intermediate spatial scale.

Decoding accuracies. The decoding accuracies of the ROIs are summarized in Figure 5. For non-symbolic magnitudes, the classifier was able to discriminate between the different numbers of dots better than chance level in all the ROIs tested, except for the visual word form area.

The SVM results for symbolic magnitudes were, however, different. We observed significant decoding accuracy for classifying symbols in the left superior parietal lobule, inferior occipital cortex, fusiform gyrus, right superior frontal gyrus. However, we did not find a significant decoding accuracy in the IPS and in its different subdivisions: left anterior IPS, right anterior IPS, left posterior IPS and right posterior IPS. Together with the results on a large spatial scale, these results suggests that in order to discriminate between the neural patterns of symbols one needs a lot of information which seems to be distributed throughout the brain. 


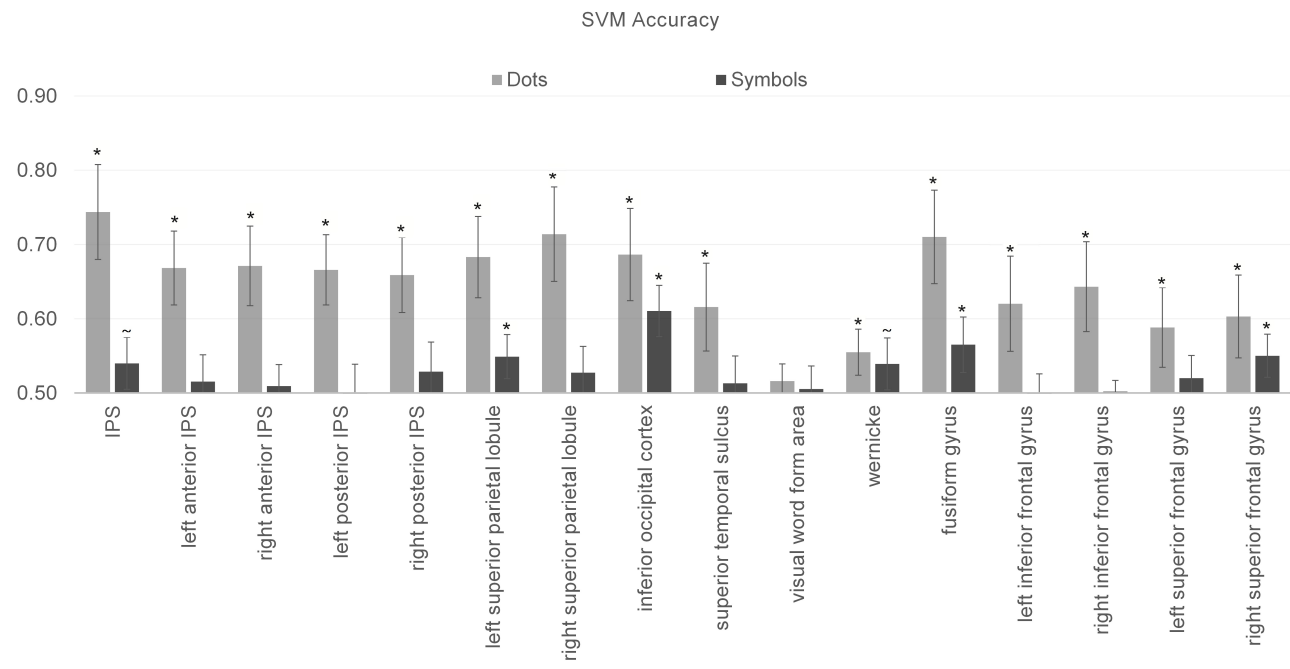

Figure 5: Significant decoding performances for symbolic and non-symbolic stimuli for ROIs on an intermediate spatial scale. The error bars represent the standard error of mean (* significant with FDR-correction $(q=0.05)$ for multiple comparisons; $\sim$ significant if no FDR-correction is applied).

Neural distance effect. Results for the neural distance effect of symbolic and non-symbolic magnitudes are summarized in Figure 6. For non-symbolic magnitudes, most regions had a significant distance effect: IPS $\left(R^{2}=0.12\right.$, $p<0.001)$, left anterior IPS $\left(R^{2}=0.06, p<0.02\right)$, right anterior IPS $\left(R^{2}=0.05, p<0.02\right)$, left posterior IPS $\left(R^{2}=0.12, p<0.001\right)$, right posterior IPS $\left(R^{2}=0.19, p<0.001\right)$, left superior parietal lobule $\left(R^{2}=\right.$ $0.16, p<0.001)$, right superior parietal lobule $\left(R^{2}=0.24, p<0.001\right)$, inferior occipital cortex $\left(R^{2}=0.11, p<0.001\right)$, superior temporal sulcus $\left(R^{2}=0.05, p=0.03\right)$, fusiform gyrus $\left(R^{2}=0.16, p<0.001\right)$, left inferior frontal gyrus $\left(R^{2}=0.11, p<0.001\right)$, right inferior frontal gyrus $\left(R^{2}=0.09\right.$, $p<0.001)$ and right superior frontal gyrus $\left(R^{2}=0.09, p=0.01\right)$. Only two ROIs that showed significant decoding accuracy, did not demonstrate a significant distance effect: Wernickes area $\left(R^{2}=0.02, p=0.21\right)$ and the left superior frontal gyrus $\left(R^{2}=0.01, p=0.38\right)$. This suggests that the significant decoding of non-symbolic magnitudes in these two areas was not related to magnitude.

For symbolic magnitudes, there was no distance effect in any of the ROIs that showed significant decoding accuracy: left superior parietal lobule $\left(R^{2}=\right.$ 
0.003, $p=0.62)$, inferior occipital cortex $\left(R^{2}=0.0003, p=0.86\right), p=$ $0.13)$, fusiform gyrus $\left(R^{2}=0.02, p=0.16\right)$ and right superior frontal gyrus $\left(R^{2}=0.01, p=0.30\right)$. Thus, we did not find any magnitude related activity in the ROIs tested on a large and intermediate spatial scale for the symbolic magnitudes.

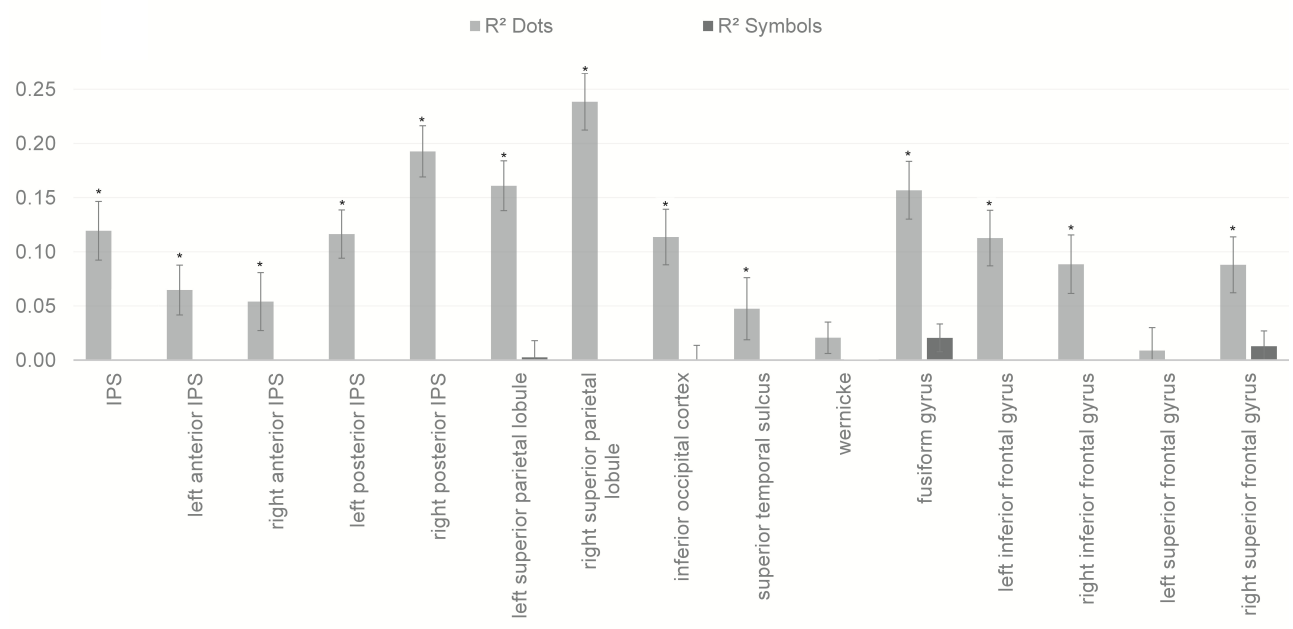

Figure 6: Significant decoding performances for symbolic and non-symbolic stimuli for ROIs on an intermediate spatial scale. The error bars represent the standard error of mean $(*$ significant with FDR-correction $(q=0.05)$ for multiple comparisons).

Association digits and dots. We again tested the neural representational overlap between symbolic and non-symbolic magnitudes in the abovementioned ROIs by applying generalization SVM. Similar to the large scale analyses, we did not find any generalization from symbolic to non-symbolic magnitudes or from non-symbolic to symbolic magnitudes.

\subsubsection{Results on a small spatial scale: searchlight analysis (SLA)}

A searchlight analysis is ideal for finding where on a local spatial scale (a) the neural patterns for stimuli in symbolic and non-symbolic formats are distinguishable (b) where the neural patterns of both formats represent a magnitude and (c) if there is any overlap between symbolic and non-symbolic representations.

We applied a whole-brain SLA with a radius of twice the voxel size resulting in a searchlight cluster of 33 voxels. This analysis resulted in two maps with 
decoding accuracy (symbolic and non-symbolic magnitudes), two maps with the distance effect (symbolic and non-symbolic magnitudes) and two maps with generalization accuracy (from symbolic to non-symbolic and from nonsymbolic to symbolic magnitudes) for each subject. These resulting maps were submitted to a group analysis and corrected for multiple comparisons (Figure 7).

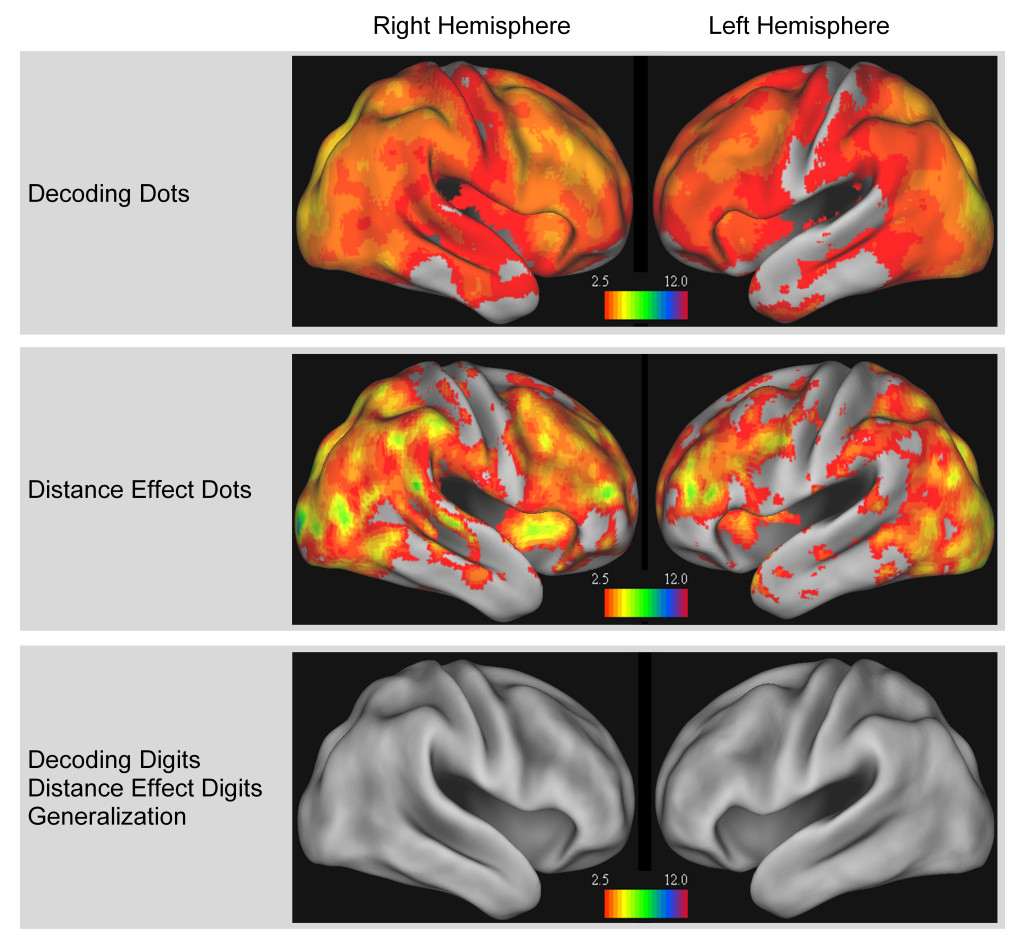

Figure 7: Results of the whole-brain searchlight analysis for FDR-corrected threshold of $\mathrm{t}=2.5$.

Decoding SLA. For both symbolic and non-symbolic magnitudes the SLA confirmed the results from the decoding analyses. The neural representations of non-symbolic magnitudes seemed to be present throughout the brain and on a very local spatial scale. For the symbolic magnitudes, we observed no representational information on a local spatial scale, which suggests that the relatively low decoding accuracies in the ROI analysis relied on the inclusion of a large number of individually defined voxels. 
Neural distance effect. The SLA for the neural distance effects of nonsymbolic magnitudes revealed that in most regions with significant decoding accuracies there was a neural distance effect. This results corresponds to the ROI-based neural distance effect analysis. For symbolic magnitudes, the SLA did not reveal any magnitude related activity on a local spatial scale in the cortex, also confirming the ROI-based neural distance effect analysis.

Association digits and dots. Concerning the ROI-based generalization analysis, the SLA also confirmed the absence of an abstract representation on a local spatial scale as there was no generalization from symbolic to nonsymbolic and from non-symbolic to symbolic magnitudes.

\subsubsection{Correlations between behavioral and imaging data}

It has been suggested that reaction time differences between conditions in a comparison task are correlated with the brain activity of those conditions (Pinel et al., 2001b), suggesting an effect of task difficulty on brain activity. We therefore correlated the difference (symbolic non-symbolic magnitudes) in reaction time between formats with the difference in decoding between formats. This was done for the All Regions and parietal cortex, because we had no a priori hypothesis for the specific area where this correlation would emerge. In All Regions there was a significant negative correlation $(r=-0.51, p=0.04)$ while in the parietal cortex a non-significant negative correlation was observed $(r=-0.45, p=0.08)$. As these correlations are negative, pointing to higher decoding when the reaction is longer, they do not reflect effects of task difficulty, but rather indicate how much attention and processing was needed by the subject to deal with the stimuli.

We have also correlated the behavioral distance effect (based on reaction times, as explained above) with the neural distance effect in All Regions and parietal cortex. This correlation was significant for non-symbolic magnitudes $(r=0.51, p=0.046)$ in All Regions and not significant $(r=0.16, p=$ $0.553)$ in the parietal cortex. We found no significant correlation between the behavioral distance effect and the neural distance effect of symbolic magnitudes in All Regions $(r=0.30, p=0.27)$ and parietal cortex $(r=$ $0.18, p=0.50)$. The latter could be explained by recourse to the failure to observe a neural distance effect for symbolic magnitudes in the All Regions and parietal cortex.

\section{Discussion}

The present study was conducted to identify neural correlates underlying symbolic and non-symbolic magnitude processing. In contrast to the 
existing body of studies that mainly used univariate fMRI methods to probe this question, we applied MVPA to unravel the neural activity patterns of numerical magnitudes. To the best of our knowledge, only two studies have applied this technique before, i.e. Eger et al. (2009) and Damarla \& Just (2012). However, these two studies restricted the focus of their analyses to the entire parietal cortex without examining any smaller regions of interest inside and outside the parietal cortex. Extending these two studies, we applied MVPA outside the parietal cortex and ran these analyses on three spatial scales: a large scale (the entire cortex, frontal cortex, parietal cortex, temporal cortex and occipital cortex), an intermediate scale with 16 ROIs throughout the whole cortex and a local scale by applying a whole brain searchlight analysis.

In summary, we found different neural patterns for non-symbolic compared to symbolic magnitudes. For dot arrays, most brain regions showed a neural distance effect, indicating a widespread representation of numerical magnitude for dot arrays. By contrast, symbolic magnitudes were only distinguishable when entire lobules were used as ROIs, and in some smaller ROIs (left superior parietal lobule, inferior occipital cortex, fusiform gyrus and right superior frontal gyrus), albeit with lower decoding accuracy. This pattern of results indicated that the neural representations of symbolic magnitudes were widely distributed across the entire cortex. No evidence was found for a neural distance effect of symbolic magnitudes in our data. The generalization analyses revealed no overlapping representations for digits and dots in any of the selected regions of interest. Results from the ROI-based MVPA analysis were extended by a whole-brain searchlight analysis, which yielded the same results. In all, these data are very hard to reconcile with an abstract representation of numerical magnitude.

\subsection{Representations of symbolic and non-symbolic magnitudes are not re- stricted to only the parietal cortex}

In contrast to previous MVPA studies that limited their scope to the parietal cortex, the present study is the first study to apply MVPA to the entire cortex, the individual lobes and 16 ROIs for decoding symbolic and non-symbolic magnitudes. Concerning the parietal cortex, we found significant decoding performances for both symbols and dot arrays, which is in accordance with Eger et al. (2009) and Damarla \& Just (2012). However, we also observed significant decoding for both formats in the frontal, occipital and temporal cortices, suggesting the presence of neural representation of symbolic and non-symbolic magnitudes on a large spatial scale in the entire cortex. Our results indicate that only looking at the parietal cortex shows 
a small part of the story. Because there are no previous numerical cognition MVPA fMRI studies looking at other ROIs besides the entire parietal cortex, we cannot directly compare the current results with previous MVPA data.

The existing univariate and adaptation fMRI studies pinpointed the IPS as the number module in the brain, against the background of similar neural effects for symbolic and non-symbolic magnitudes (Dehaene et al., 2003; Fias et al., 2003, Piazza et al., 2007). We found significant decoding accuracies for dots in the IPS, suggesting the presence of distinctive neural patterns for non-symbolic magnitudes in the IPS. However, this was not observed for symbolic magnitudes.

More crucially, we also applied MVPA to other ROIs besides the IPS and outside the parietal cortex. Our results show the decoding of both formats outside the IPS, with significant decoding performances for dots in regions in the frontal, occipital and temporal cortices. For symbolic stimuli, we observed significant decoding in the fusiform gyrus, right superior frontal gyrus, left superior parietal lobule, and inferior occipital cortex. This suggests that the neural representations of symbolic and non-symbolic magnitudes are not restricted to the IPS or the parietal cortex.

In this context caution is needed when interpreting the nature of these representations across the entire brain. It is most likely that the representations of dots and symbols in, for example, the inferior occipital cortex are more related to the visual properties of the stimuli than to their numerosity. This is in line with (Gebuis \& Reynvoet, 2012a) who showed that the comparison of (non-symbolic) number also includes many non-numerical processing (e.g., perceptual, action, semantics). The parietal cortex, on the other hand, can be expected to contribute more than the other regions to representing magnitudes and numerositiy, while occipital regions are known to represent the visual properties of stimuli and prefrontal cortex might process taskrelated aspects (e.g. process small and large numbers differently). These non-numerical representations will probably contribute the most to the nonsymbolic numbers because there is little decoding in the symbolic numbers across the ROIs.

\subsection{Representations of dots and digits are different}

Our findings suggest a divergence in the neural representations between symbolic and non-symbolic stimuli. First, in all the lobes we observed higher decoding performance for non-symbolic stimuli compared to symbolic stimuli. This higher decoding accuracy for dots versus digits was also found in 
the parietal cortex by Eger et al. (2009) and Damarla \& Just (2012). Because we extended these two studies by not restricting MVPA to the entire parietal cortex, the current data indicate that the higher decoding performance for dots versus digits is seen not only in the parietal cortex, but also in the other lobes.

A possible explanation for this finding might be that the symbols (digits) share more features than dot patterns. Although being different in magnitude, the symbol ' 2 ' and ' 8 ' share many visual characteristics, such as being one visual symbol and being processed in the same way (reading). In contrast, these visual characteristics are very different between 2 and 8 dots, and these dot patterns might processed in different ways: 2 dots are processed in an exact way, whereas 8 dots might be approximated.

The different decoding results for symbolic and non-symbolic magnitudes could be explained in the light of recent findings by Shum et al. (2013). They used intracranial electrophysiological recordings in epileptic patients and reported higher activation for Arabic digits compared with letters, false fonts, number words and non-number words in the inferior temporal gyrus. Their study suggested one location that was very specific for processing Arabic digits. However, this regions falls in the fMRI signal-dropout zone, thus we cannot capture the functional response for symbolic magnitudes in this region with fMRI.

Although we cannot exclude the possibility of a symbolic number region outside the range of fMRI, we still found significant decoding accuracies for symbolic magnitudes. This was not observed in one small region, but it was more scattered throughout the entire cortex, suggesting that the neural representations of digits are embedded on a large spatial scale. This finding is in line with Eger et al. (2009) who reported the absence of neural representations for symbolic magnitudes on a relatively local spatial scale in the parietal cortex in contrast to distinguishable neural patterns on a large spatial scale in the parietal cortex.

In accordance with the studies of Eger et al. (2009), Damarla \& Just (2012) and Shum et al. (2013), the present study suggests different processing in different locations of non-symbolic and symbolic stimuli. These neuroimaging studies converge nicely with previous behavioral studies showing differences between both formats. For example, Lyons et al. (2012) demonstrated that cross-format number comparisons were more difficult than within-format number comparisons, which suggests that symbolic and non-symbolic magnitudes are independently processed. 
4.3. The neural distance effect for symbolic and non-symbolic magnitudes is different

To test whether the decoding performances found in the ROIs were reflecting a magnitude representation, we verified whether a neural distance effect could be found in these regions. In line with previous studies, we observed the presence of a neural distance effect for non-symbolic magnitudes and an absence of this effect for symbolic magnitudes in the parietal cortex (Damarla \& Just, 2012; Eger et al., 2009). We extended our scope to ROIs in the parietal, frontal, temporal and occipital cortices and found magnitude representations for dot arrays in most ROIs. However, we did not find magnitude representations for symbols in any of our ROIs (large and intermediate spatial scale), including the IPS, even though the number comparison task demanded an explicit magnitude judgment. This suggests that, in contrast to non-symbolic magnitudes the neural activation in the IPS for symbolic magnitudes is not related to the underlying magnitude.

Despite this differences in the neural distance effects of dots and digits, we observed a behavioral distance effect for both symbols and dots. However, the mere presence of a behavioral distance effect does not necessarily indicate similar underlying cognitive processes in dealing with symbolic and non-symbolic stimuli. For example, Lyons et al. (2012) showed that in the presence of behavioral distance effects for digits and dots in a number comparison task, format number comparisons were more difficult, which suggests different cognitive processes for symbolic and non-symbolic magnitudes. Also, Campbell (1994); Dehaene \& Akhavein (1995); Ganor-Stern \& Tzelgov (2008) observed interactions between numerical distance and format, indicating that different cognitive processes are involved in symbolic and non-symbolic tasks. Maloney et al. (2010) observed no correlation between the distance effect of dots and symbols, suggesting different underlying mechanisms for both formats. In line with this, the present study did not observe a correlation between the behavioral distance effect of digits and dots.

\subsection{Relationship between symbolic and non-symbolic magnitudes}

We found no overlapping neural patterns for symbolic and non-symbolic magnitudes in any of the ROIs, suggesting no representational overlap for both formats. This result contradicts the study of Eger et al. (2009), which found a generalization from digits to dots $(57 \%)$, but no generalization from dots to digits. Eger et al. (2009) explained these results in the context of a neural network model constructed by Verguts \& Fias (2004). This network model suggests that a subset of neurons that originally responds to 
non-symbolic magnitudes acquires selectivity to the corresponding symbolic number and, while preserving response selectivity to non-symbolic magnitudes, becomes more narrowly tuned to the specific symbolic number.

However, Damarla \& Just (2012) were not able to replicate the data by Eger et al. (2009) and reported that their poor generalization across formats suggests that the neural representation of numerical magnitudes in the parietal areas is primarily format-specific. On top of that, Eger et al. (2009) did not find any generalization in their parietal cortex searchlight analysis. In line with this, we also did not find any generalization in the whole brain searchlight analysis. Note that our study is very similar to the study of Eger et al. (2009), and that our study had an appropriate sensitivity (we were able to decode each of the two formats) and reasonable power (16 subjects). Against this background, it seems appropriate to conclude that no detectable overlapping representations between symbolic and non-symbolic magnitudes are present, neither in the parietal cortex nor in the cortex as whole.

\subsection{Whole-brain searchlight analysis}

This study is the first to apply a whole brain searchlight analysis in the context of decoding symbolic and non-symbolic magnitudes. With this analysis, we aimed to detect a neural distance effect for both formats, and tested the representational overlap between digits and dots. The whole brain searchlight analysis resulted in an accurate decoding and neural distance effect for dots in most parts of the cortex, which suggests a very local representation of non-symbolic magnitude in the brain. A small spherical region at many cortical positions is sufficient to decode the number of dots that is shown to the participants. For the symbolic stimuli no significant decoding and neural distance effect on a local scale was found. Together with the ROI-based MVPA analysis, this suggests that symbolic stimuli are represented in a very distributed manner across the entire brain and need the inclusion of a large number of individually defined voxels to detect their neural pattern.

\subsection{Is there an abstract representation of magnitude in the brain?}

The majority of the studies concerning numerical cognition have suggested that a domain-specific number module is present in the IPS Ansari et al., 2006; Cohen Kadosh et al., 2005, Eger et al., 2003; Fias et al., 2003; Piazza et al., 2007; Pinel et al., 2004). Although the results of these studies are seen as evidence for the domain-specificity of the IPS, Shuman \& Kanwisher (2004) challenged the conclusions drawn from these studies based on 
three fMRI experiments. These authors did not find an adaptation effect for repeated numerosities in different formats, but observed less strong activations for number tasks than for color tasks in the IPS. They also showed that the IPS responded more strongly to difficult compared to easy tasks with no differences between number tasks and color tasks. Hence, the experiments of Shuman \& Kanwisher (2004) do not support the hypothesis of the IPS as a domain-specific area for abstract number processing.

Turning to our study, if such abstract representation would exist one should find: (a) similar decoding results for both symbolic and non-symbolic magnitudes, (b) similar neural distance effects for both formats and (c) a representational overlap between symbols and dot arrays. In contrast to this, we found (a) different decoding results for digits and dots with major differences for the location of neural representations for both formats; (b) differences in the presence of the neural distance effects between digits and dots; and (c) no generalization effects from digits to dots and vice versa, which indicates no representational overlap between dots and digits. This pattern of findings was observed on a large spatial scale (entire cortices), an inter-mediate spatial scale (regions of interest) and a small spatial scale (whole-brain searchlight with a radius of twice the voxel size) and is hard to reconcile with the existence of an abstract magnitude representation.

\section{Conclusion}

We confirm the conclusion of Eger et al. (2009) that MVPA allows one to distinguish the representations of different numerical magnitudes with a higher accuracy for dot patterns than for symbols. However, in our study the numerical representations were format-dependent without any common format-independent representation of magnitude: Classifiers trained on dot patterns were not able to generalize to symbols (or vice versa). Although subjects were performing a magnitude-related task showing a distance effect at the behavioral level, the MVPA results showed a distance effect for nonsymbolic but not for symbolic stimuli. The searchlight analyses did not reveal any overlapping representations between both formats anywhere in the cortex. These findings are hard to reconcile with the idea of an abstract representation of numerical magnitudes.

\section{Acknowledgements}

We would like to thank the reviewers, Annelies Baeck, Nicky Daniels and Lien Peters for their helpful comments on this paper. This work was 
supported by the Fund for Scientific Research Flanders by a fellowship to J.B. and by a IDO Project by the KU Leuven (ZKC3232).

\section{References}

Ansari, D. (2008). Effects of development and enculturation on number representation in the brain. Nature reviews. Neuroscience, 9, 278-291. URL: http://www.ncbi.nlm.nih.gov/pubmed/18334999, doi:10.1038/ nrn2334.

Ansari, D., Dhital, B., \& Siong, S. C. (2006). Parametric effects of numerical distance on the intraparietal sulcus during passive viewing of rapid numerosity changes. Brain research, 1067, 181-188. URL: http://www . ncbi.nlm.nih.gov/pubmed/16359648, doi:10.1016/j.brainres.2005. 10.083 .

Barth, H., Kanwisher, N., \& Spelke, E. (2003). The construction of large number representations in adults. Cognition, 86, 201-221. URL: http://linkinghub.elsevier.com/retrieve/pii/ S0010027702001786, doi:10.1016/S0010-0277(02)00178-6.

Brainard, D. H. (1997). The Psychophysics Toolbox. Spatial Vision, 10, 433-436. URL: http://booksandjournals.brillonline.com/content/ 10.1163/156856897x00357. doi:10.1163/156856897X00357

Brannon, E. M., Wusthoff, C. J., Gallistel, C., \& Gibbon, J. (2001). Numerical Subtraction in the Pigeon: Evidence for a Linear Subjective Number Scale. Psychological Science, 12, 238-243. URL: http://pss.sagepub. com/lookup/doi/10.1111/1467-9280.00342. doi:10.1111/1467-9280. 00342 .

Buckley, P. B., \& Gillman, C. B. (1974). Comparisons of digits and dot patterns. Journal of Experimental Psychology, 103, 1131-1136. URL: http://content.apa.org/journals/xge/103/6/1131. doi:10. 1037/h0037361.

Campbell, J. (1994). Architectures for numerical cognition. Cognition, 53, 1-44. URL: http://www.sciencedirect.com/science/article/ pii/0010027794900752.

Cappelletti, M., Lee, H. L., Freeman, E. D., \& Price, C. J. (2010). The role of right and left parietal lobes in the conceptual processing of numbers. Journal of cognitive neuroscience, 22, 
331-346. URL: http://www.pubmedcentral.nih.gov/articlerender. fcgi?artid=2808313\&tool=pmcentrez\&rendertype=abstract. doi:10 . 1162/jocn.2009.21246.

Caspers, S., Geyer, S., Schleicher, A., Mohlberg, H., Amunts, K., \& Zilles, K. (2006). The human inferior parietal cortex: cytoarchitectonic parcellation and interindividual variability. NeuroImage, 33, 430-448. URL: http://www.ncbi.nlm.nih.gov/pubmed/16949304, doi:10.1016/ j.neuroimage.2006.06.054.

Cohen Kadosh, R., Henik, A., Rubinsten, O., Mohr, H., Dori, H., van de Ven, V., Zorzi, M., Hendler, T., Goebel, R., \& Linden, D. E. J. (2005). Are numbers special? The comparison systems of the human brain investigated by fMRI. Neuropsychologia, 43, 1238-1248. URL: http://www.ncbi.nlm.nih.gov/pubmed/15949508, doi:10.1016/ j.neuropsychologia.2004.12.017.

Cohen Kadosh, R., \& Walsh, V. (2009). Numerical representation in the parietal lobes: abstract or not abstract? The Behavioral and brain sciences, 32, 313-373. URL: http://www.ncbi.nlm.nih.gov/pubmed/ 19712504, doi:10.1017/S0140525X09990938.

Damarla, S. R., \& Just, M. A. (2012). Decoding the representation of numerical values from brain activation patterns. Human brain mapping, 00 . URL: http://www.ncbi.nlm.nih.gov/pubmed/22505340, doi:10.1002/ hbm.22087.

De Smedt, B., \& Gilmore, C. K. (2011). Defective number module or impaired access? Numerical magnitude processing in first graders with mathematical difficulties. Journal of experimental child psychology, 108, 278-292. URL: http://www.ncbi.nlm.nih.gov/pubmed/20974477. doi:10.1016/j.jecp.2010.09.003.

De Smedt, B., Noël, M., Gilmore, C., \& Ansari, D. (). How do symbolic and non-symbolic numerical magnitude processing relate to individual differences in children's mathematical skills? A review of evidence from brain and behavior. Trends in Neuroscience \& Education, . doi:10.1016/ j.tine.2013.06.001.

Dehaene, S. (1992). Varieties of numerical abilities. Cognition, 44, 1-42.

Dehaene, S. (2009). The case for a notation-independent representation of number. Behavioral and Brain Sciences, 32, 333. URL: http:// 
Www. journals . cambridge.org/abstract_S0140525X09990033, doi:10. 1017/S0140525X09990033.

Dehaene, S., \& Akhavein, R. (1995). Attention, automaticity, and levels of representation in number processing. Journal of Experimental Psychology: Learning, Memory, and Cognition, 21, 314-326. doi:10.1037/ 10278-7393.21.2.314.

Dehaene, S., \& Cohen, L. (1997). Cerebral pathways for calculation: double dissociation between rote verbal. Cortex, 33, 219-250.

Dehaene, S., Dupoux, E., \& Mehler, J. (1990). Is numerical comparison digital? Analogical and symbolic effects in two-digit number comparison. Journal of Experimental Psychology: Human Perception and Performance, 16, 626-641. URL: http://doi.apa.org/getdoi.cfm?doi=10. 1037/0096-1523.16.3.626. doi:10.1037/0096-1523.16.3.626.

Dehaene, S., Izard, V., \& Piazza, M. (2005). Control over non-numerical parameters in numerosity experiments. Unpublished manuscript (available at www.unicog.org), .

Dehaene, S., Piazza, M., Pinel, P., \& Cohen, L. (2003). Three parietal circuits for number processing. Cognitive neuropsychology, 20, 487-506. URL: http://www.ncbi.nlm.nih.gov/pubmed/20957581. doi:10.1080/ 02643290244000239 .

Eger, E., Michel, V., Thirion, B., Amadon, A., Dehaene, S., \& Kleinschmidt, A. (2009). Deciphering cortical number coding from human brain activity patterns. Current biology : CB, 19, 1608-1615. URL: http://www.ncbi. nlm.nih.gov/pubmed/19781939, doi:10.1016/j.cub.2009.08.047.

Eger, E., Sterzer, P., Russ, M. O., Giraud, A.-L., \& Kleinschmidt, A. (2003). A supramodal number representation in human intraparietal cortex. Neuron, 37, 719-725. URL: http://www.ncbi.nlm.nih.gov/ pubmed/12597867.

Feigenson, L., Dehaene, S., \& Spelke, E. (2004). Core systems of number. Trends in cognitive sciences, 8, 307-314. URL: http://www.ncbi.nlm. nih.gov/pubmed/15242690, doi:10.1016/j.tics.2004.05.002.

Fias, W., Lammertyn, J., Caessens, B., \& Orban, G. a. (2007). Processing of abstract ordinal knowledge in the horizontal segment of the intraparietal sulcus. The Journal of neuroscience : the official journal of the 
Society for Neuroscience, 27, 8952-6. URL: http://www.ncbi.nlm.nih. gov/pubmed/17699676, doi:10.1523/JNEUROSCI . 2076-07.2007.

Fias, W., Lammertyn, J., Reynvoet, B., Dupont, P., \& Orban, G. a. (2003). Parietal representation of symbolic and nonsymbolic magnitude. Journal of cognitive neuroscience, 15, 47-56. URL: http://www.ncbi.nlm.nih. gov/pubmed/12590842, doi:10.1162/089892903321107819.

Ganor-Stern, D., \& Tzelgov, J. (2008). Across-notation automatic numerical processing. Journal of experimental psychology. Learning, memory, and cognition, 34, 430-7. URL: http://www.ncbi.nlm.nih.gov/ pubmed/18315418, doi:10.1037/0278-7393.34.2.430.

Gebuis, T., \& Reynvoet, B. (2012a). Continuous visual properties explain neural responses to nonsymbolic number. Psychophysiology, 49, 16491659. URL: http://www.ncbi.nlm.nih.gov/pubmed/23046492, doi:10. 1111/j.1469-8986.2012.01461.x.

Gebuis, T., \& Reynvoet, B. (2012b). The interplay between nonsymbolic number and its continuous visual properties. Journal of experimental psychology. General, 141, 642-648. URL: http://www.ncbi.nlm.nih. gov/pubmed/22082115, doi:10.1037/a0026218.

Gerardi, K., Goette, L., \& Meier, S. (2013). Numerical ability predicts mortgage default. Proceedings of the National Academy of Sciences, (pp. 15). URL: http://www.pnas.org/cgi/doi/10.1073/pnas.1220568110. doi:10.1073/pnas.1220568110.

Gilmore, C., Attridge, N., Clayton, S., Cragg, L., Johnson, S., Marlow, N., Simms, V., \& Inglis, M. (2013). Individual differences in inhibitory control, not non-verbal number acuity, correlate with mathematics achievement. PloS one, 8, e67374. URL: http://www.pubmedcentral.nih.gov/articlerender. fcgi?artid=3681957\&tool=pmcentrez\&rendertype=abstract. doi:10. 1371/journal.pone.0067374.

Göbel, S. M., Johansen-Berg, H., Behrens, T., \& Rushworth, M. F. S. (2004). Response-selection-related parietal activation during number comparison. Journal of cognitive neuroscience, 16, 1536-1551. URL: http://www. ncbi.nlm.nih.gov/pubmed/15601517. doi:10.1162/0898929042568442. 
Holloway, I. D., \& Ansari, D. (2010). Developmental specialization in the right intraparietal sulcus for the abstract representation of numerical magnitude. Journal of cognitive neuroscience, 22, 2627-37. URL: http://www.ncbi.nlm.nih.gov/pubmed/19929327, doi:10.1162/jocn. 2009.21399,

Holloway, I. D., Battista, C., Vogel, S. E., \& Ansari, D. (2013). Semantic and Perceptual Processing of Number Symbols: Evidence from a Crosslinguistic fMRI Adaptation Study. Journal of cognitive neuroscience, 25, 388-400. URL: http://www.ncbi.nlm.nih.gov/pubmed/23163414. doi:10.1162/jocn \_a\_00323.

Ischebeck, A., Heim, S., Siedentopf, C., Zamarian, L., Schocke, M., Kremser, C., Egger, K., Strenge, H., Scheperjans, F., \& Delazer, M. (2008). Are numbers special? Comparing the generation of verbal materials from ordered categories (months) to numbers and other categories (animals) in an fMRI study. Human brain mapping, 29, 894-909. URL: http: //www.ncbi.nlm.nih.gov/pubmed/17705220, doi 10.1002/hbm.20433.

Iuculano, T., Tang, J., Hall, C. W. B., \& Butterworth, B. (2008). Core information processing deficits in developmental dyscalculia and low numeracy. Developmental science, 11, 669-680. URL: http://www.ncbi.nlm.nih. gov/pubmed/18801122, doi $10.1111 / \mathrm{j} .1467-7687.2008 .00716 . \mathrm{x}$.

Jaffe-Katz, A., Budescu, D. V., \& Wallsten, T. S. (1989). Timed magnitude comparisons of numerical and nonnumerical expressions of uncertainty. Memory \& Cognition, 17, 249-264. URL: http://www.springerlink. com/index/10.3758/BF03198463, doi:10.3758/BF03198463.

Kriegeskorte, N., \& Bandettini, P. (2007). Analyzing for information, not activation, to exploit high-resolution fMRI. NeuroImage, 38, 649-662. URL: http://www.pubmedcentral.nih.gov/articlerender. fcgi?artid=2099257\&tool=pmcentrez\&rendertype=abstract. doi:10. 1016/j.neuroimage.2007.02.022.

Kriegeskorte, N., Goebel, R., \& Bandettini, P. (2006). Informationbased functional brain mapping. PNAS, 103, 3863-3868. URL: $\quad$ http://www.pubmedcentral.nih.gov/articlerender. fcgi?artid=1383651\&tool=pmcentrez\&rendertype=abstract. doi:10.1073/pnas.0600244103. 
Landerl, K., \& Kölle, C. (2009). Typical and atypical development of basic numerical skills in elementary school. Journal of experimental child psychology, 103, 546-565. URL: http://www.ncbi.nlm.nih.gov/pubmed/ 19254797, doi:10.1016/j.jecp.2008.12.006.

Lee, Y.-S., Janata, P., Frost, C., Hanke, M., \& Granger, R. (2011). Investigation of melodic contour processing in the brain using multivariate patternbased fMRI. NeuroImage, 57, 293-300. URL: http://www.ncbi.nlm. nih.gov/pubmed/21315158, doi:10.1016/j.neuroimage.2011.02.006.

Lipkus, I. M., \& Peters, E. (2009). Understanding the role of numeracy in health: proposed theoretical framework and practical insights. Health education \& behavior : the official publication of the Society for Public Health Education, 36, 106581. URL: http://www.pubmedcentral.nih.gov/articlerender.fcgi? artid=2783983\&tool=pmcentrez\&rendertype=abstract. doi:10.1177/ 1090198109341533 .

Lonnemann, J., Linkersdörfer, J., Hasselhorn, M., \& Lindberg, S. (2011). Symbolic and non-symbolic distance effects in children and their connection with arithmetic skills. Journal of Neurolinguistics, 24, 583-591. URL: http://linkinghub.elsevier.com/retrieve/pii/ S0911604411000297, doi:10.1016/j.jneuroling.2011.02.004.

Lyons, I. M., \& Ansari, D. (2009). The cerebral basis of mapping nonsymbolic numerical quantities onto abstract symbols: an fMRI training study. Journal of cognitive neuroscience, 21, 1720-35. URL: http://www.ncbi. nlm.nih.gov/pubmed/18823231, doi:10.1162/jocn.2009.21124.

Lyons, I. M., Ansari, D., \& Beilock, S. L. (2012). Symbolic estrangement: evidence against a strong association between numerical symbols and the quantities they represent. Journal of experimental psychology. General, 141, 635-641. URL: http://www.ncbi.nlm.nih.gov/pubmed/22329752. doi:10.1037/a0027248.

Maldjian, J., Laurienti, P., Burdette, J., \& Kraft, R. (2003). An Automated Method for Neuroanatomic and Cytoarchitectonic Atlas-based Interrogation of fMRI Data Sets. NeuroImage, 19, 1233-1239.

Maloney, E. a., Risko, E. F., Preston, F., Ansari, D., \& Fugelsang, J. (2010). Challenging the reliability and validity of cognitive measures: the case of the numerical distance effect. Acta psychologica, 
134, 154-61. URL: http://www.ncbi.nlm.nih.gov/pubmed/20185118. doi:10.1016/j.actpsy.2010.01.006.

Maruyama, M., Pallier, C., Jobert, A., Sigman, M., \& Dehaene, S. (2012). The cortical representation of simple mathematical expressions. NeuroImage, 61, 1444-1460. URL: http://www.ncbi.nlm.nih.gov/pubmed/ 22521479, doi:10.1016/j.neuroimage.2012.04.020.

Moyer, R., \& Bayer, R. (1976). Mental comparison and the symbolic distance effect. Cognitive Psychology, 8, 228-246. URL: http:// linkinghub.elsevier.com/retrieve/pii/0010028576900256. doi:10. 1016/0010-0285(76)90025-6.

Moyer, R., \& Landauer, T. (1967). Time required for Judgements of Numerical Inequality. Nature, 215, 1519-1520. URL: http://www.nature. com/doifinder/10.1038/2151519a0, doi 10.1038/2151519a0.

Naccache, L., \& Dehaene, S. (2001). Unconscious semantic priming extends to novel unseen stimuli. Cognition, 80, 215-29. URL: http://www.ncbi. nlm.nih.gov/pubmed/11274983.

Nelson, W., Reyna, V. F., Fagerlin, A., Lipkus, I., \& Peters, E. (2008). Clinical implications of numeracy: theory and practice. Annals of behavioral medicine : a publication of the Society of Behavioral Medicine, 35, 261-74. URL: http://www.ncbi.nlm.nih.gov/pubmed/18677452. doi: $10.1007 / \mathrm{s} 12160-008-9037-8$.

Nieder, A., \& Dehaene, S. (2009). Representation of number in the brain. Annual review of neuroscience, 32, 185-208. URL: http://www.ncbi. nlm.nih.gov/pubmed/19400715. doi:10.1146/annurev.neuro.051508. 135550 .

Nieder, A., Freedman, D. J., \& Miller, E. K. (2002). Representation of the quantity of visual items in the primate prefrontal cortex. Science (New York, N.Y.), 297, 1708-1711. URL: http://www.ncbi.nlm.nih. gov/pubmed/12215649, doi:10.1126/science.1072493.

Nieder, A., \& Miller, E. K. (2003). Coding of cognitive magnitude: compressed scaling of numerical information in the primate prefrontal cortex. Neuron, 37, 149-157. URL: http://www.ncbi.nlm.nih.gov/pubmed/ 12526780. 
Norman, K. a., Polyn, S. M., Detre, G. J., \& Haxby, J. V. (2006). Beyond mind-reading: multi-voxel pattern analysis of fMRI data. Trends in cognitive sciences, 10, 424-30. URL: http://www.ncbi.nlm.nih.gov/ pubmed/16899397, doi:10.1016/j.tics.2006.07.005.

Op de Beeck, H. P., Brants, M., Baeck, A., \& Wagemans, J. (2010). Distributed subordinate specificity for bodies, faces, and buildings in human ventral visual cortex. NeuroImage, 49, 3414-3425. URL: http://www.ncbi.nlm.nih.gov/pubmed/19922804, doi:10.1016/ j.neuroimage.2009.11.022.

van Opstal, F., \& Verguts, T. (2011). The origins of the numerical distance effect: The samedifferent task. Journal of Cognitive Psychology, 23, 112-120. URL: http://www.tandfonline.com/doi/abs/10.1080/ 20445911.2011.466796, doi:10.1080/20445911.2011.466796.

Piazza, M., Izard, V., Pinel, P., Le Bihan, D., \& Dehaene, S. (2004). Tuning curves for approximate numerosity in the human intraparietal sulcus. Neuron, 44, 547-555. doi:10.1016/j.neuron.2004.10.014.

Piazza, M., Pinel, P., Le Bihan, D., \& Dehaene, S. (2007). A magnitude code common to numerosities and number symbols in human intraparietal cortex. Neuron, 53, 293-305. URL:http://www.ncbi.nlm.nih.gov/ pubmed/17224409. doi:10.1016/j.neuron.2006.11.022.

Pinel, P., Dehaene, S., Rivière, D., \& LeBihan, D. (2001a). Modulation of parietal activation by semantic distance in a number comparison task. NeuroImage, 14, 1013-1026. doi:10.1006/nimg.2001.0913.

Pinel, P., Dehaene, S., Rivière, D., \& LeBihan, D. (2001b). Modulation of parietal activation by semantic distance in a number comparison task. NeuroImage, 14, 1013-1026. URL: http://www.ncbi.nlm.nih. gov/pubmed/11697933, doi 10.1006/nimg.2001.0913.

Pinel, P., Piazza, M., Le Bihan, D., \& Dehaene, S. (2004). Distributed and overlapping cerebral representations of number, size, and luminance during comparative judgments. Neuron, 41, 983-993. URL: http://www . ncbi.nlm.nih.gov/pubmed/15046729.

Polk, T. a., Reed, C. L., Keenan, J. M., Hogarth, P., \& Anderson, C. a. (2001). A dissociation between symbolic number knowledge and analogue magnitude information. Brain and cognition, 47, 545-563. URL: 
http://www.ncbi.nlm.nih.gov/pubmed/11748908, doi:10.1006/brcg. 2001.1486.

Raizada, R. D. S., Tsao, F.-M., Liu, H.-M., \& Kuhl, P. K. (2010). Quantifying the adequacy of neural representations for a cross-language phonetic discrimination task: prediction of individual differences. Cerebral cortex (New York, N.Y. : 1991), 20, 112. URL: http://www.pubmedcentral.nih.gov/articlerender.fcgi? artid=2860710\&tool=pmcentrez\&rendertype=abstract. doi:10.1093/ cercor/bhp076.

Reyna, V. F., Nelson, W. L., Han, P. K., \& Dieckmann, N. F. (2009). How numeracy influences risk comprehension and medical decision making. Psychological bulletin, 135, 94373. URL: http://www.pubmedcentral.nih.gov/articlerender. fcgi?artid=2844786\&tool=pmcentrez\&rendertype=abstract. doi:10.1037/a0017327.

Roggeman, C., Santens, S., Fias, W., \& Verguts, T. (2011). Stages of nonsymbolic number processing in occipitoparietal cortex disentangled by fMRI adaptation. The Journal of neuroscience, 31, 7168-7173. URL: http://www.ncbi.nlm.nih.gov/pubmed/21562280, doi:10.1523/ JNEUROSCI . 4503-10.2011.

Rousselle, L., \& Noël, M.-P. (2007). Basic numerical skills in children with mathematics learning disabilities: a comparison of symbolic vs non-symbolic number magnitude processing. Cognition, 102, 361-395. URL: http://www.ncbi.nlm.nih.gov/pubmed/16488405. doi:10.1016/ j.cognition.2006.01.005.

Santens, S., Roggeman, C., Fias, W., \& Verguts, T. (2010). Number processing pathways in human parietal cortex. Cerebral cortex (New York, N.Y. : 1991), 20, 77-88. URL: http://www.pubmedcentral.nih.gov/ articlerender.fcgi?artid=2792188\&tool=pmcentrez\&rendertype= abstract. doi:10.1093/cercor/bhp080.

Sawamura, H., Shima, K., \& Tanji, J. (2002). Numerical representation for action in the parietal cortex of the monkey. Nature, 415, 918-922. URL: http://www.ncbi.nlm.nih.gov/pubmed/11859371. doi:10.1038/ 415918a. 
Sekuler, R., \& Mierkiewicz, D. (1977). Children's judgments of numerical inequality. Child Development, 48, 630-633. Child development, 48, 630633 .

Shepard, R. N., Kilpatric, D. W., \& Cunningham, J. P. (1975). The internal representation of numbers. Cognitive Psychology, 7, 82-138. URL: http://linkinghub.elsevier.com/retrieve/pii/ 0010028575900067, doi:10.1016/0010-0285(75)90006-7.

Shum, J., Hermes, D., Foster, B. L., Dastjerdi, M., Rangarajan, V., Winawer, J., Miller, K. J., \& Parvizi, J. (2013). A brain area for visual numerals. The Journal of neuroscience : the official journal of the Society for Neuroscience, 33, 6709-6715. doi:10.1523/JNEUROSCI .4558-12.2013.

Shuman, M., \& Kanwisher, N. (2004). Numerical magnitude in the human parietal lobe; tests of representational generality and domain specificity. Neuron, 44, 557-569. URL: http://www.ncbi.nlm.nih.gov/pubmed/ 15504334. doi:10.1016/j.neuron.2004.10.008.

Verguts, T., \& Fias, W. (2004). Representation of number in animals and humans: a neural model. Journal of cognitive neuroscience, 16, 14931504. URL: http://www.ncbi.nlm.nih.gov/pubmed/15601514. doi 10 . 1162/0898929042568497.

Walther, D. B., Caddigan, E., Fei-fei, L., \& Beck, D. M. (2009). Natural Scene Categories Revealed in Distributed Patterns of Activity in the $\mathrm{Hu}-$ man Brain, . 29, 10573-10581. doi:10.1523/JNEUROSCI.0559-09.2009.

Zago, L., Pesenti, M., Mellet, E., Crivello, F., Mazoyer, B., \& TzourioMazoyer, N. (2001). Neural correlates of simple and complex mental calculation. NeuroImage, 13, 314-327. URL: http://www.ncbi.nlm.nih. gov/pubmed/11162272, doi $10.1006 /$ nimg.2000.0697.

Zhang, H., Chen, C., \& Zhou, X. (2012). Neural correlates of numbers and mathematical terms. NeuroImage, 60, 230-240. URL: http://www.ncbi. nlm.nih.gov/pubmed/22202882, doi:10.1016/j.neuroimage.2011.12. 006.

Zikmund-Fisher, B. J., Smith, D. M., Ubel, P. A., \& Fagerlin, A. (2007). Validation of the Subjective Numeracy Scale: Effects of Low Numeracy on Comprehension of Risk Communications and Utility Elicitations. Medical Decision Making, 27, 663-671. URL: http://mdm.sagepub.com/cgi/ doi/10.1177/0272989X07303824, doi $10.1177 / 0272989$ X07303824. 
Zorzi, M., Di Bono, M. G., \& Fias, W. (2011). Distinct representations of numerical and non-numerical order in the human intraparietal sulcus revealed by multivariate pattern recognition. NeuroImage, 56, 674-680. URL: http://www.ncbi.nlm.nih.gov/pubmed/20600989, doi:10.1016/ j.neuroimage.2010.06.035 